\title{
Bisphosphonates in oncology: evidence for the prevention of skeletal events in patients with bone metastases
}

\author{
Thomas J Polascik \\ Division of Urology, Department \\ of Surgery, Duke University Medical \\ Center, Durham, NC, USA
}

\begin{abstract}
Bone metastases frequently occur in patients with advanced solid tumors, particularly breast and prostate cancers, and nearly all patients with multiple myeloma have some degree of skeletal involvement. The strides made in treating these primary tumors have extended median survival times and thereby increased patient risk for skeletal-related events (SREs), including pathologic fractures, spinal cord compression, need for palliative radiation therapy or surgery to bone, and hypercalcemia. Bisphosphonates, inhibitors of osteoclastic bone resorption that were first established as treatment of osteoporosis, have been shown to prevent and/or delay SREs related to malignancy. The results of a large, randomized phase 3 study comparing zoledronic acid and pamidronate in breast cancer or multiple myeloma patients with osteolytic lesions showed that the incidence of SREs, time to first SRE, and risk of developing a SRE were similar between treatment groups. However, in patients with solid tumors (excluding breast or prostate cancer) metastatic to the bone, only zoledronic acid has demonstrated clinical efficacy. Although bone turnover marker levels, such as N-telopeptide of type I collagen, have been shown to correlate with clinical response, additional studies are needed to validate their ability to predict response to bisphosphonate therapy.
\end{abstract}

Keywords: bisphosphonates, prevention, skeletal-related events, bone metastases, cancer

\section{Introduction}

Osteoporosis, a skeletal condition common in postmenopausal women and aging men, is characterized by low bone mass, destruction of bone microarchitecture, and increased bone turnover resulting in decreased bone strength and consequent susceptibility to fractures. ${ }^{1,2}$ Osteoporotic fractures, such as fractures of the hip, vertebral body, and distal forearm, may lead to decreased quality of life (QOL), disability, and possibly death. In the last decade, bisphosphonates, compounds that inhibit osteoclastic bone resorption, have been the most significant contribution to the advancement in osteoporosis treatment; clinical trials have demonstrated a reduction in vertebral fractures of $40 \%$ to $50 \%$ and nonvertebral fractures (including hip fractures) of $20 \%$ to $40 \%$. ${ }^{1,3}$ Bisphosphonates approved by the United States Food and Drug Administration (FDA) for the prevention and/or treatment of osteoporosis include alendronate, alendronate plus vitamin D, ibandronate, risedronate, risedronate with calcium, and zoledronic acid. ${ }^{4-10}$ Because their bioavailability is quite low, oral agents usually require daily or weekly administration (with the exception of ibandronate, which may be administered monthly) that can contribute to low patient compliance rates. ${ }^{6-9}$ Intravenous (IV) bisphosphonates may be administered less frequently (eg, on a monthly, quarterly, or yearly basis). ${ }^{6,10-12}$ In general, patient compliance rates with prescribed IV bisphosphonate regimens are higher than with oral bisphosphonates. 
In addition to osteoporosis, bisphosphonates have been used to prevent and/or treat cancer-related bone complications. ${ }^{3,13}$ Patients who develop bone metastases are at increased risk for developing skeletal-related events (SREs), such as intractable bone pain requiring opioid analgesics or palliative radiation therapy, pathologic fractures, spinal cord compression, a need for surgery, and hypercalcemia of malignancy (HCM). ${ }^{14}$ SREs are a consequence of excessive bone metabolism, primarily bone resorption, which characterizes malignant bone lesions. ${ }^{3}$ Local bone pain requiring radiation therapy and pathologic fractures are the most commonly reported SREs. ${ }^{3}$

As a result of advancements in the primary treatment of several solid tumors and hematologic malignancies, patients are surviving longer, placing them at an increased risk for developing bone metastasis and SREs that may complicate their clinical course, adversely affect QOL, and increase medical costs. ${ }^{14-16}$ Bone metastases are particularly prevalent in patients with advanced metastatic breast or prostate cancers, affecting approximately $70 \%$ of patients. ${ }^{3}$ Although observed less frequently, bone metastases also occur in patients with lung, kidney, and thyroid tumors. ${ }^{17}$ Nearly all patients with advanced multiple myeloma (MM) develop bone involvement during the course of their disease since this malignancy colonizes in the bone marrow. ${ }^{14,18}$

\section{Metastatic bone disease}

Under normal circumstances, bone homeostasis is achieved through balanced resorption of old bone by osteoclasts and formation of new bone by osteoblasts. ${ }^{19}$ Metastatic bone disease alters the normal bone remodeling process by causing osteolytic bone destruction and abnormal osteoblastic bone formation, often with one process more dominant than the other, resulting in an imbalance in normal bone homeostasis. ${ }^{18-20}$ Although historically bone metastases from breast cancer or MM have been characterized as osteolytic lesions and prostate cancer bone metastases have been primarily osteoblastic in nature, recent evidence suggest that both bone processes are present in many patients. ${ }^{18,20}$ Without bisphosphonate treatment, it is estimated that patients with bone metastases from advanced cancer will experience, on average, 2 to 4 SREs per year. ${ }^{17}$ Thus, bone complications of cancer are a considerable clinical concern, and preventing or delaying the occurrence of such events is an important treatment objective. Although palliation has traditionally been the primary goal of therapy, the introduction of bisphosphonates has afforded oncologists with an effective therapeutic option for preventing and/or treating SREs associated with bone metastases.

\section{Mechanism of action of bisphosphonates}

Because of their ability to diminish bone resorption and subsequently normalize calcium levels, prevent development of new osteolytic lesions, and reduce the risk of fractures, bisphosphonates are the treatment of choice for skeletal complications of malignancy. ${ }^{21}$ Bisphosphonates are pyrophosphate analogs that preferentially bind to bone at sites of active metabolism, are released from the bone matrix during bone resorption, and inhibit osteoclast activity and survival. ${ }^{3,21}$ Variable side chains determine the potency and side effect profile of each agent. ${ }^{21}$ These compounds can be grouped into two classes according to their chemical structure and molecular mechanism of action (Figure 1). ${ }^{22}$ The newer nitrogen (N)-containing, second- or third-generation compounds, including alendronate, ibandronate, pamidronate, risedronate, and zoledronic acid, inhibit the enzyme farnesyl diphosphate synthase in the cholesterol mevalonate pathway and thereby suppress osteoclast-mediated bone resorption, whereas the non - N-containing, first-generation bisphosphonates, such as clodronate, etidronate, and tiludronate, induce osteoclast apoptosis via metabolism into cytotoxic analogues of adenosine 5'-triphosphate. ${ }^{21,23,24}$ The N-containing agents are more potent than the non-N-containing bisphosphonates, inhibiting bone resorption at micromolar concentrations. ${ }^{3}$ Only IV zoledronic acid and IV pamidronate are approved by the FDA for cancer-related indications..$^{25,26}$ In Europe, oral clodronate, IV pamidronate, and oral and IV ibandronate have received regulatory approval for patients with bone metastases secondary to breast cancer. ${ }^{3,27}$ Only zoledronic acid has received US and European approval for the treatment of bone metastases independent of the primary tumor type..$^{25,28}$

\section{Evaluating efficacy of bisphosphonates}

Developing composite end points of similar clinical significance may be appropriate when the clinical benefit of a drug is multifaceted as is the case with bisphosphonates. ${ }^{29}$ Using a SRE as a quantifiable clinical end point was first applied to studies assessing pamidronate for prevention of SREs. This end point included one or more of the following: pathologic fracture, radiation therapy for local pain, surgery to stabilize near-fractures, or spinal cord compression. Subsequently, SREs were used as the primary end points for most of the trials assessing a bisphosphonate for this indication. However, the definition or names for SREs have differed slightly between studies (see Tables $1-4$ ), sometimes being 


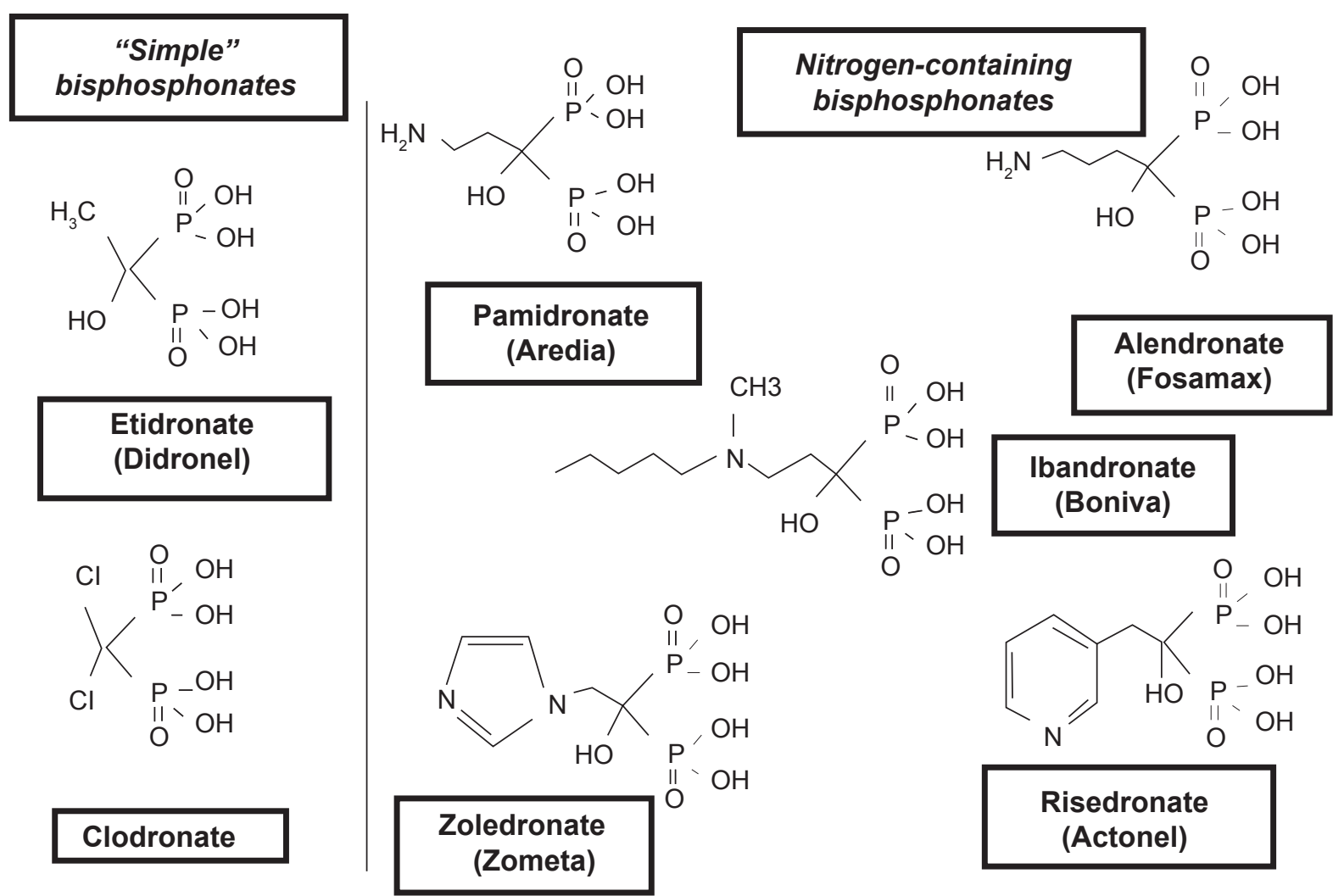

Figure I The structure of simple and nitrogen-containing bisphosphonates. Reprinted with permission from Russell RG. Bisphosphonates: mode of action and pharmacology. Pediatrics. 2007; I 19:SI50-S162.22 Copyright @ by the AAP.

referred to as skeletal complications or bone events. ${ }^{30-55}$ Furthermore, HCM has been excluded from the definition of a SRE in some trials because bisphosphonates have been shown to be effective for the treatment of HCM before studies investigating bisphosphonates as preventive therapy for SREs were developed. ${ }^{31,56}$

Several clinical studies designed to evaluate bisphosphonates to prevent skeletal complications have demonstrated clinical benefit. This article reviews the results of clinical studies assessing bisphosphonates as prevention and/or treatment for cancer-related bone complications in a variety of tumor types.

\section{Clinical studies}

\section{Breast cancer}

Clinical trial results show that bisphosphonates reduce the occurrence of skeletal complications in patients with breast cancer and bone metastases (Table 1). ${ }^{30-42}$ Based on the results of two randomized, placebo-controlled clinical studies in patients with osteolytic bone metastases from breast cancer being treated with either chemotherapy or hormonal therapy, IV pamidronate was approved by the FDA for preventing SREs. ${ }^{35,37}$ Pamidronate $(90 \mathrm{mg}$ administered IV over
2-4 hours q 3-4 weeks) significantly prolonged the time to the first SRE and reduced the overall incidence of SREs for up to 2 years (see Table 1). ${ }^{35-38}$ In another placebo-controlled trial of breast cancer patients with at least one osteolytic lesion, zoledronic acid significantly lowered the risk of SREs by $39 \%$ $(p=0.027)$, reduced the proportion of patients experiencing a SRE at 1 year by $20 \%(29.8 \%$ vs $49.6 \%, p=0.003)$, and significantly prolonged the time to first SRE excluding HCM (median not reached vs 364 days, $\mathrm{p}=0.007$ ). ${ }^{33}$ Only one study has directly compared zoledronic acid with pamidronate (see Table 1)..$^{30,31}$ This large, randomized phase 3 study was designed to demonstrate the equivalence of zoledronic acid and pamidronate in reducing the incidence of SREs in patients with breast cancer or MM. Among the breast carcinoma stratum, the overall incidence of SREs other than HCM was comparable between the two study groups. ${ }^{31}$ The median time to first SRE was also similar; however, in patients receiving hormonal therapy for breast cancer, zoledronic acid $4 \mathrm{mg}$ IV significantly delayed the time to first SRE (415 vs 370 days; $\mathrm{p}=0.047$ ). Oral and IV ibandronate have also been evaluated in breast cancer patients with metastatic bone disease; compared with placebo, both formulations of ibandronate (6 $\mathrm{mg}$ IV and $50 \mathrm{mg}$ oral) have significantly reduced the 


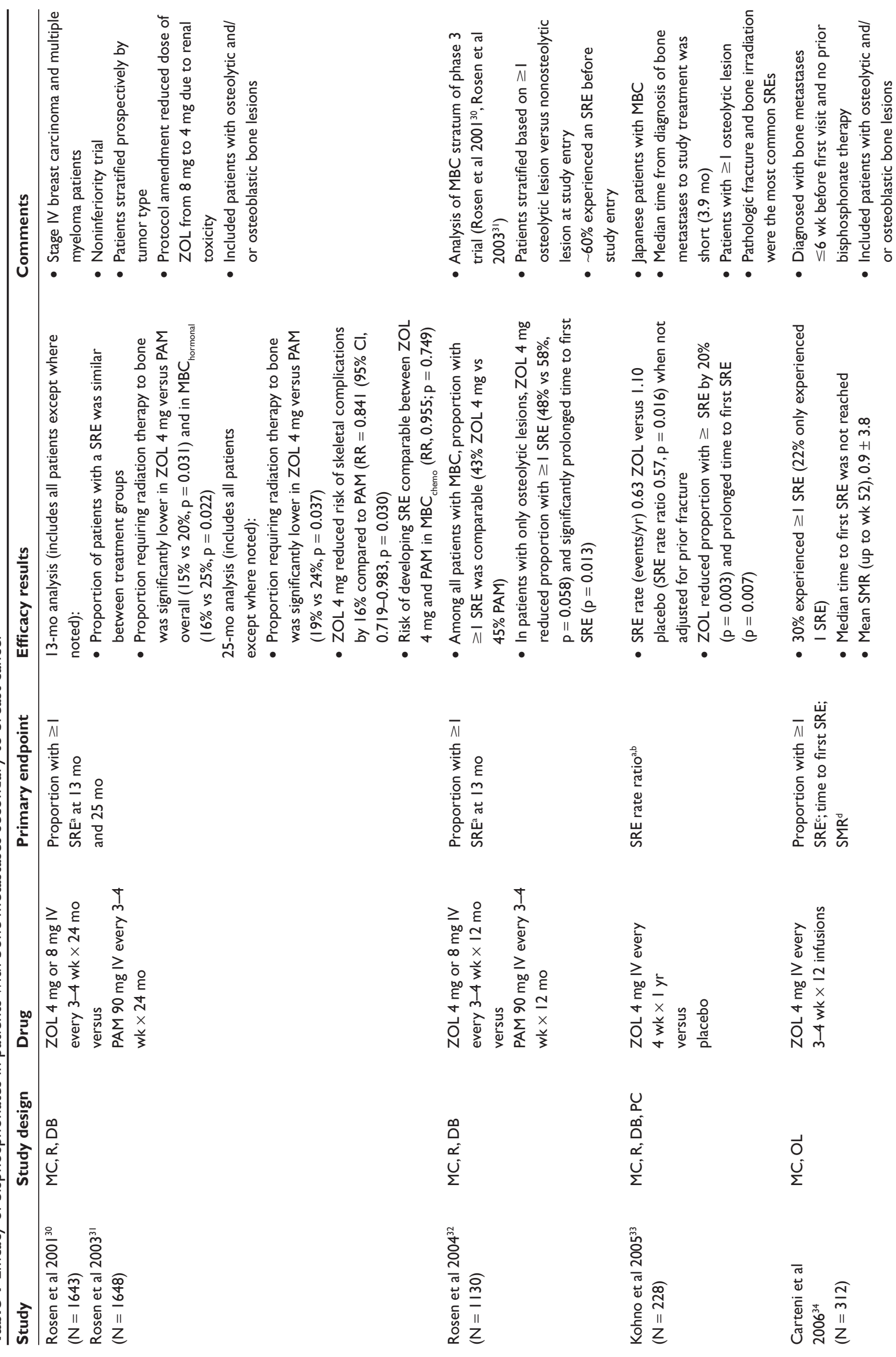



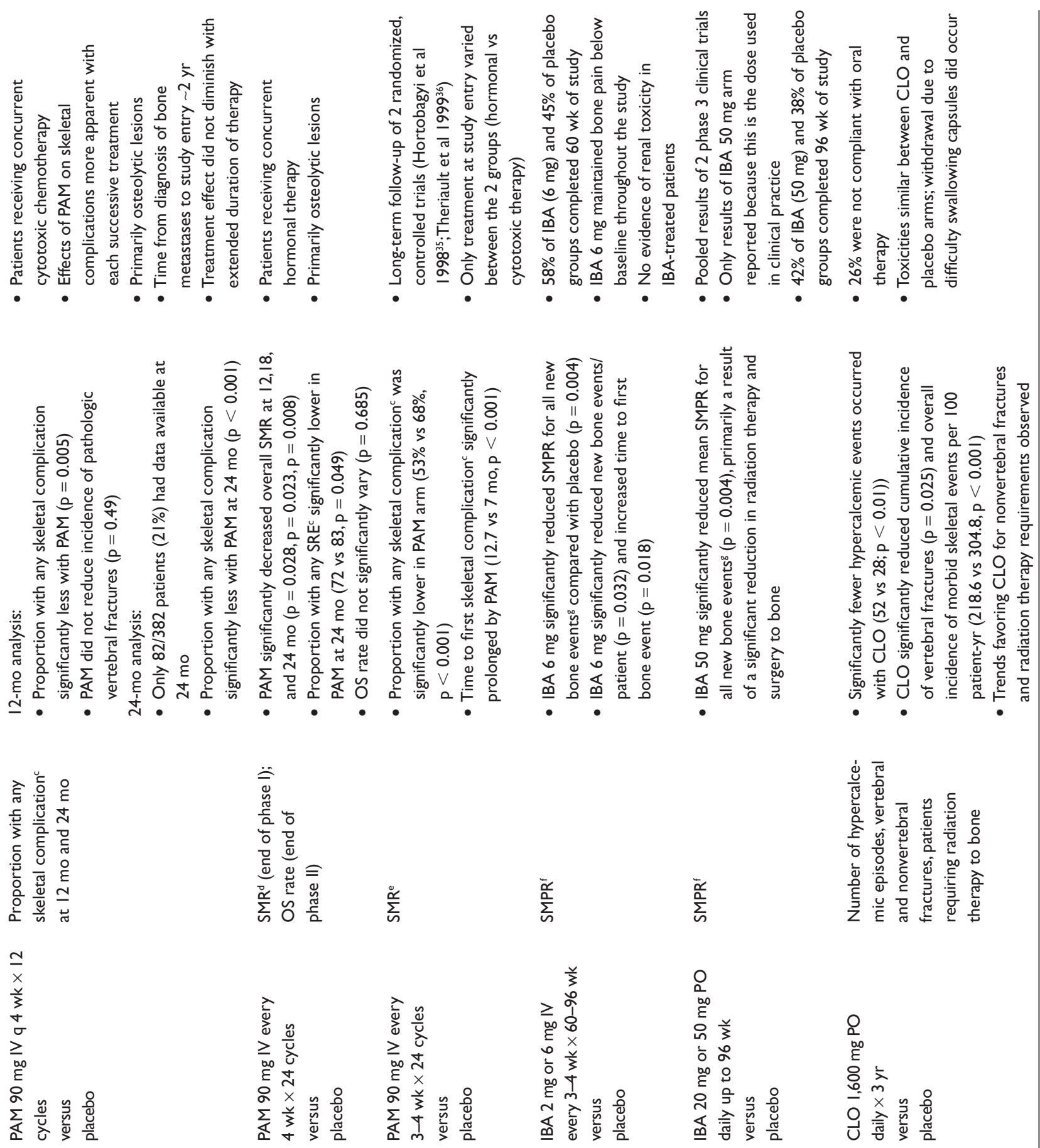

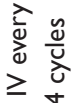

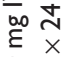

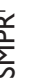
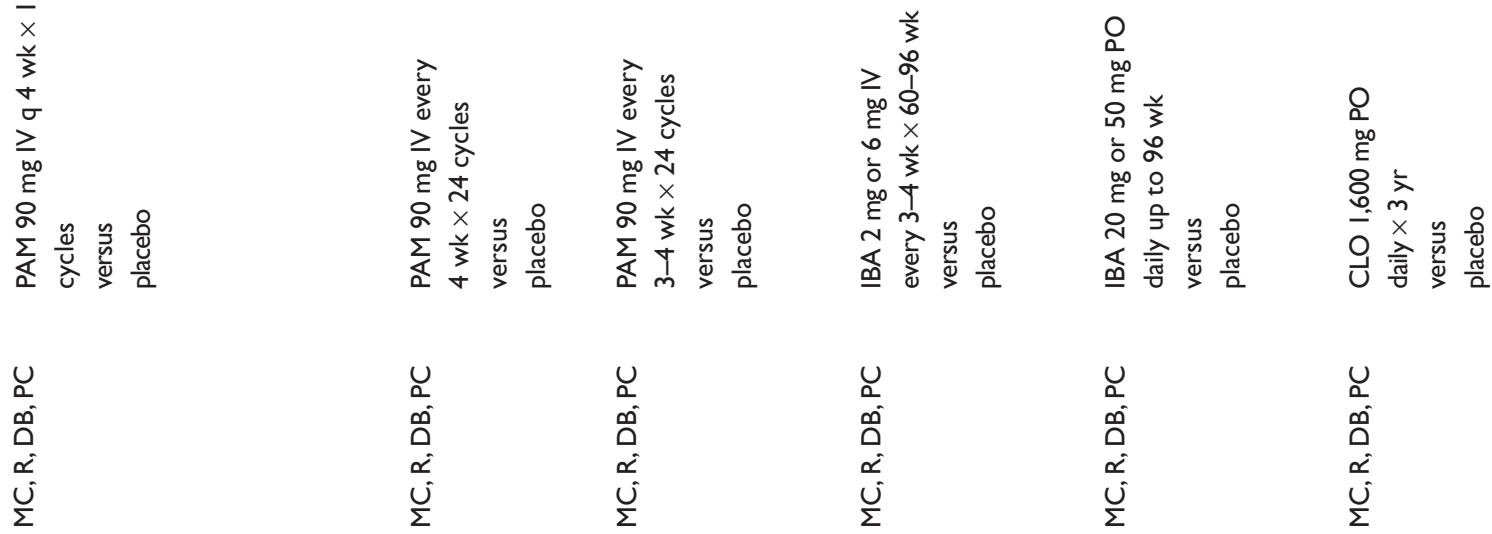

$u$
$\dot{0}$
$\stackrel{0}{a}$
$\dot{u}$
$\dot{u}$

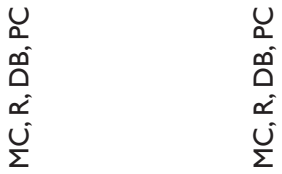
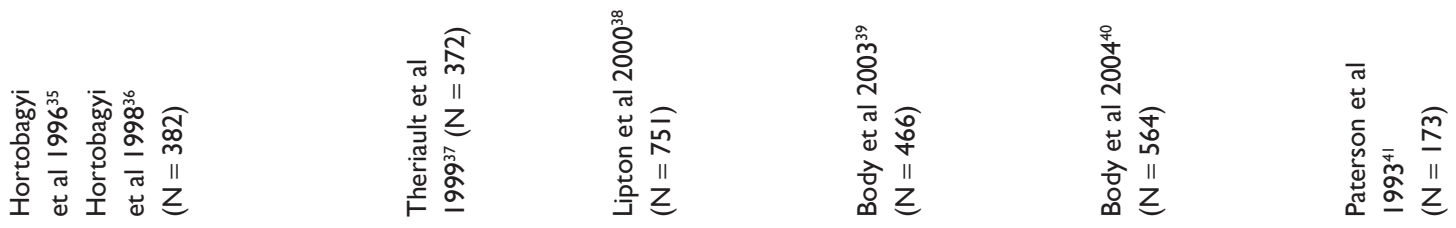
proportion of patients with an on-study SRE and delayed the time to first SRE (see Table 1). ${ }^{39,40}$ Oral clodronate also appears to reduce the rate and delay onset of SREs, and may reduce bone pain (see Table 1). ${ }^{41,42}$ Neither of these drugs, however, has been directly compared with zoledronic acid or pamidronate in this setting.

IV bisphosphonates are recommended for the prevention of skeletal complications in breast cancer patients with bone metastases. Treatment guidelines from the American Society of Clinical Oncology (ASCO) recommend either IV pamidronate $(90 \mathrm{mg}$ ) or IV zoledronic acid (4 mg) every 3 to 4 weeks for patients with radiographic evidence of bone destruction; the panel concluded that there is insufficient evidence to recommend one bisphosphonate over the other. ${ }^{57}$ The National Comprehensive Cancer Network (NCCN) recommends either IV zoledronic acid or IV pamidronate for patients with bone metastases secondary to breast cancer as well. ${ }^{58}$ However, the NCCN guidelines suggest that zoledronic acid may be superior to pamidronate therapy for treating osteolytic metastases from breast cancer. Neither clodronate nor ibandronate were recommended; clodronate is not commercially available in the US, and ibandronate does not have a cancer-related FDA indication. Moreover, the Cochrane Breast Cancer Review Group has reported the results of their meta-analysis of 21 randomized trials evaluating bisphosphonates; in 9 studies, bisphosphonates reduced the risk of skeletal complications by $17 \%$ compared with placebo or no bisphosphonate (relative risk [RR] 0.83; $95 \%$ confidence interval $[\mathrm{CI}], 0.78-0.89 ; \mathrm{p}=0.00001){ }^{59}$ Like the ASCO panel, the authors concluded that zoledronic acid appears to have equivalent efficacy when compared with pamidronate.

\section{Prostate cancer}

Although prostate cancer is most commonly associated with osteoblastic lesions, increased osteoclastic activity also disrupts normal bone metabolism when prostate cancer invades the skeleton. ${ }^{60}$ Thus, inhibition of bone resorption by bisphosphonates may be beneficial for osteoblastic metastases. Several bisphosphonates have been evaluated in patients with prostate cancer and metastatic bone disease, but only zoledronic acid has been shown to decrease SREs (Table 2). ${ }^{43-46}$ In a randomized study, patients with hormone-refractory prostate cancer (HRPC) were treated for up to 2 years with zoledronic acid or placebo; zoledronic acid significantly reduced the proportion of patients experiencing at least one SRE ( $38 \%$ vs $49 \%, 95 \%$ CI, $-20.2 \%$ to $-1.3 \%, \mathrm{p}=0.028$ ) and prolonged the time to the first SRE 


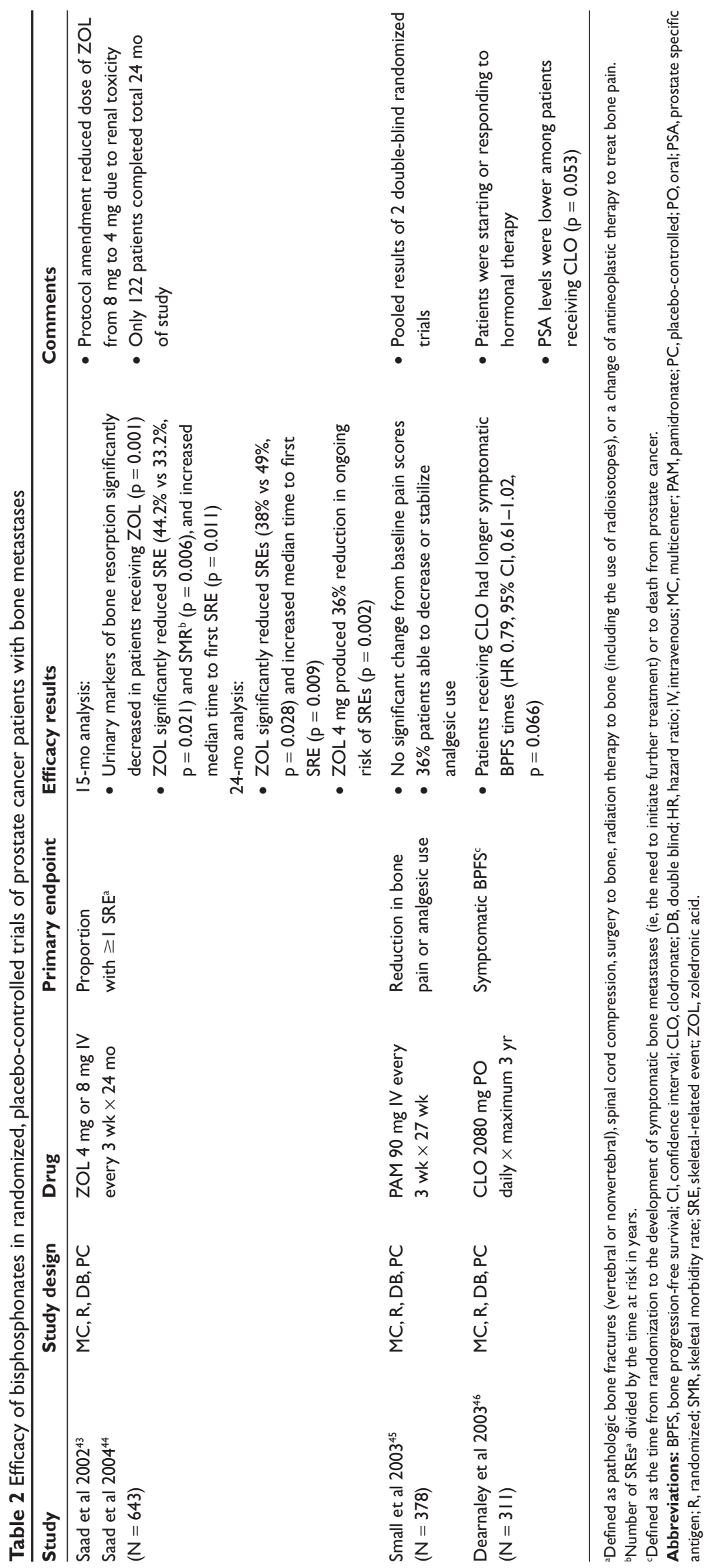




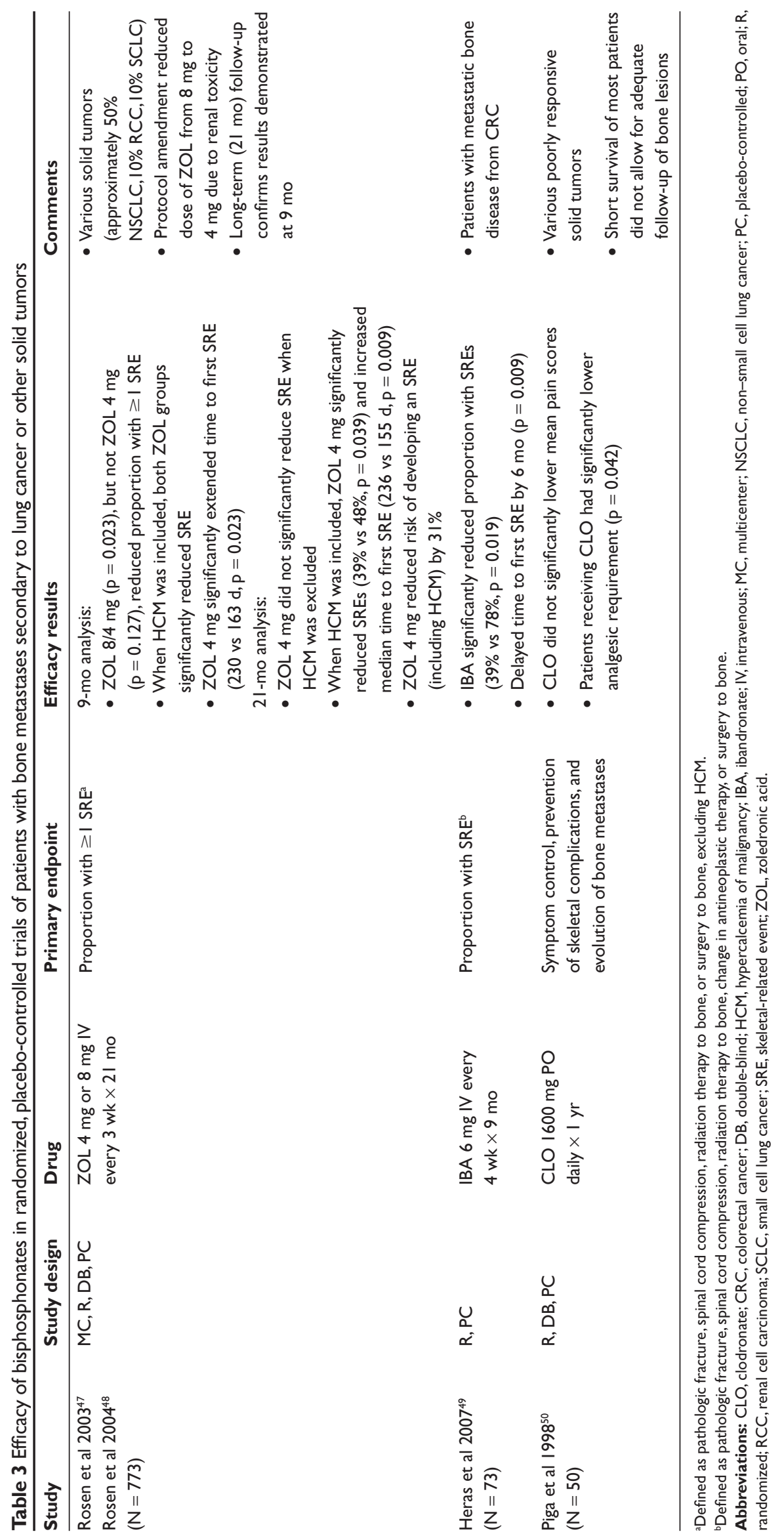


(488 vs 321 days, $\mathrm{p}=0.009$ ) compared with placebo. ${ }^{43,44}$ In this setting, pamidronate did not significantly reduce the SRE rate or reduce bone pain compared with placebo (see Table 2) ${ }^{45}$ Compared with placebo, oral clodronate $(2080 \mathrm{mg}$ daily) improved bone progression-free survival (defined as the time from randomization to the development of symptomatic bone metastases or death from prostate cancer) and the overall survival time; however, differences in these end points were not statistically significant. ${ }^{46}$ Bone progressionfree survival is similar to SRE except that it includes only symptomatic events rather than also including asymptomatic events (eg, metastasis identified on bone scan, asymptomatic vertebral fractures). The Cochrane Prostatic Diseases and Urologic Cancers Group has corroborated these findings in its meta-analysis of 10 studies evaluating bisphosphonates in metastatic prostate cancer; compared with controls, bisphosphonates reduced the risk of skeletal events by $21 \%$ (odds ratio 0.79; 95\% CI, 0.62-1.00; $\mathrm{p}=0.05$ ). ${ }^{61}$ Currently, risedronate and zoledronic acid are also being evaluated in patients with bone metastases from androgen-sensitive prostate cancer. ${ }^{62,63}$ According to consensus guidelines from the NCCN, bisphosphonate therapy should be considered for all patients with HRPC; however, the panel does not recommend a specific bisphosphonate. ${ }^{60}$

\section{Lung cancer and other solid tumors}

Only zoledronic acid $4 \mathrm{mg}$ has demonstrated significant long-term clinical benefits in patients with bone metastases from a broad range of solid tumors (Table 3). ${ }^{47-50}$ In a randomized, placebo-controlled, phase 3 study assessing patients with lung cancer or other solid tumors (excluding breast or prostate cancer), zoledronic acid $4 \mathrm{mg}$ significantly reduced the proportion of patients developing at least one SRE, including HCM at 21 months (39\% vs 48\%, p = 0.039) and delayed the onset of skeletal complications (236 vs $155 \mathrm{~d}, \mathrm{p}=0.009) .{ }^{47,48}$ Moreover, when HCM was included, zoledronic acid $4 \mathrm{mg}$ reduced the risk of developing a SRE, including $\mathrm{HCM}$, by $31 \%$ (hazard ratio, 0.693; p = 0.003) compared with placebo. ${ }^{48}$ Non - small cell lung cancer, renal cell carcinoma, and small cell lung cancer were the most common diagnoses of enrolled patients. Oral clodronate (1600 mg/day for 1 year) was also evaluated in patients with bone metastases from solid tumors poorly responsive to chemotherapy; clodronate did not significantly reduce mean pain scores compared with placebo but significantly reduced use of analgesics $(p=0.042) .{ }^{50}$ Ibandronate $6 \mathrm{mg}$ $\mathrm{IV}$, administered every 4 weeks for 9 months to patients with metastatic bone disease from colorectal cancer, significantly reduced the proportion of patients who experienced SREs (39\% vs $78 \%, \mathrm{p}=0.019)$ and delayed time to the first SRE by at least 6 months ( $>279$ vs 93 days, $p=0.009)$ compared with placebo. ${ }^{49}$ Ibandronate has not been evaluated in other solid tumors. For bone metastases related to solid tumors other than breast or prostate cancer, zoledronic acid is the only bisphosphonate that has received worldwide regulatory approval. ${ }^{25}$ Consensus guidelines for the use of bisphosphonates for patients with lung cancer or other solid tumors (except breast and prostate cancer) are not available. ${ }^{64}$

\section{Multiple myeloma}

The long-term efficacy and safety of bisphosphonate therapy for prevention of SREs in patients with advanced MM and osteolytic lesions is well established (Table 4). ${ }^{30,31,51-55}$ In a randomized, placebo-controlled trial, pamidronate (90 mg IV administered over 4 hours q 4 weeks) significantly delayed the onset $(p=0.016)$ and reduced the incidence of skeletal complications $(\mathrm{p}=0.016)$ for up to 21 months. ${ }^{51.52}$ Consequently, a large, international, randomized, phase 3 trial was designed to demonstrate equivalence (defined as difference in SRE rate of less than $8 \%$ ) between either 4 or $8 \mathrm{mg}$ zoledronic acid and standard-dose pamidronate (90 mg). ${ }^{30,31}$ Because of renal safety concerns, the protocol was amended to reduce zoledronic acid from $8 \mathrm{mg}$ to $4 \mathrm{mg} .{ }^{30}$ After 25 months of follow-up, the percentage of MM patients who developed a SRE excluding HCM (47\%, 4 mg zoledronic acid vs $51 \%$, pamidronate), the median time to first SRE including HCM (380 vs 286 days, $\mathrm{p}=0.538$ ), and the risk of developing a skeletal complication ( RR, 0.932; $\mathrm{p}=0.593$ ) were similar between the treatment groups. ${ }^{31}$ Most of these studies assessed zoledronic acid administered every 3 to 4 weeks; however, because of its long half-life and evidence supporting the use of longer dosing intervals for other indications, less frequent dosing (every $12 \mathrm{wk}$ ) is being evaluated. ${ }^{65}$ Furthermore, oral clodronate (1600 mg daily) has established its ability to significantly reduce the incidence of nonvertebral and vertebral fractures compared with placebo in MM patients. ${ }^{54}$ Long-term follow-up indicates that clodronate treatment may also prolong survival time in patients without overt vertebral fractures at diagnosis. ${ }^{55}$ Ibandronate has not been shown to reduce skeletal complications in this patient population. ${ }^{53}$

ASCO recently released an update to their clinical practice guidelines for the role of bisphosphonates in MM. ${ }^{66}$ For MM patients who have radiographic evidence of osteolytic bone destruction or spinal compression, ASCO recommends treatment with either pamidronate $90 \mathrm{mg}$ IV delivered over 
WIn

MOMn

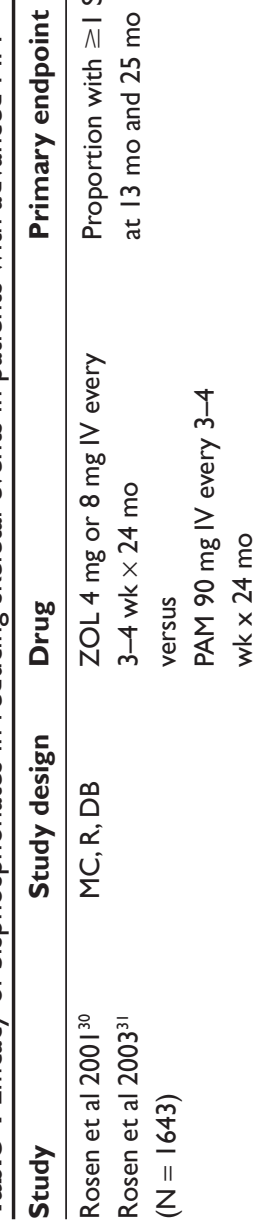

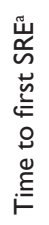
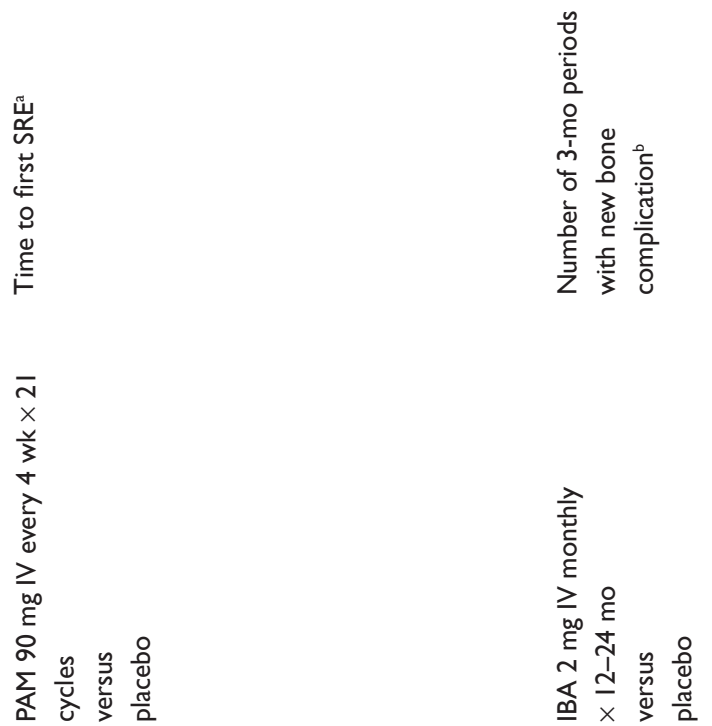

$u$
0
0
0
uे
ú

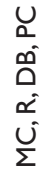

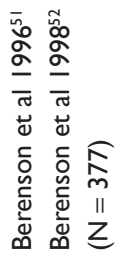

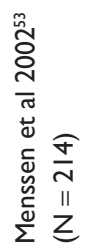



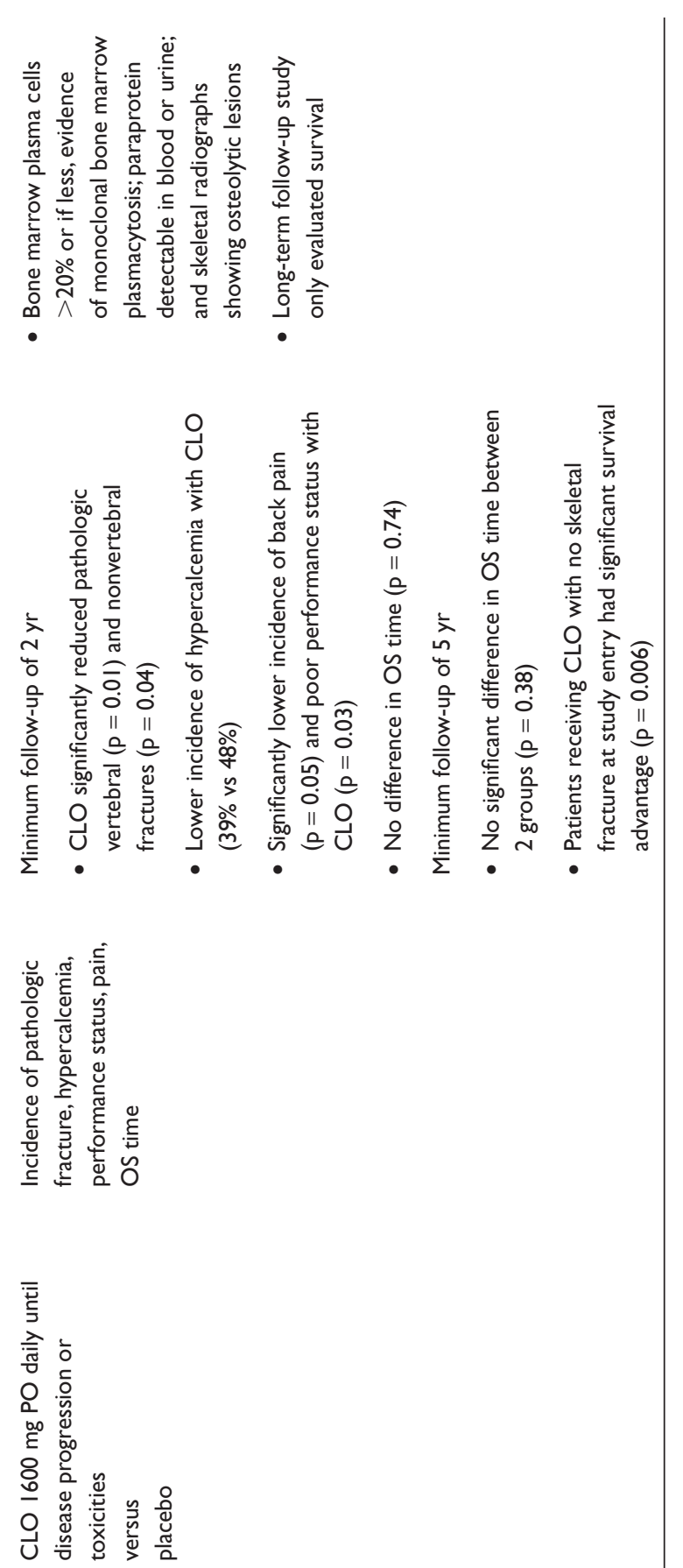

$u$
0
0
0
0
$u$
$u$
$\Sigma$
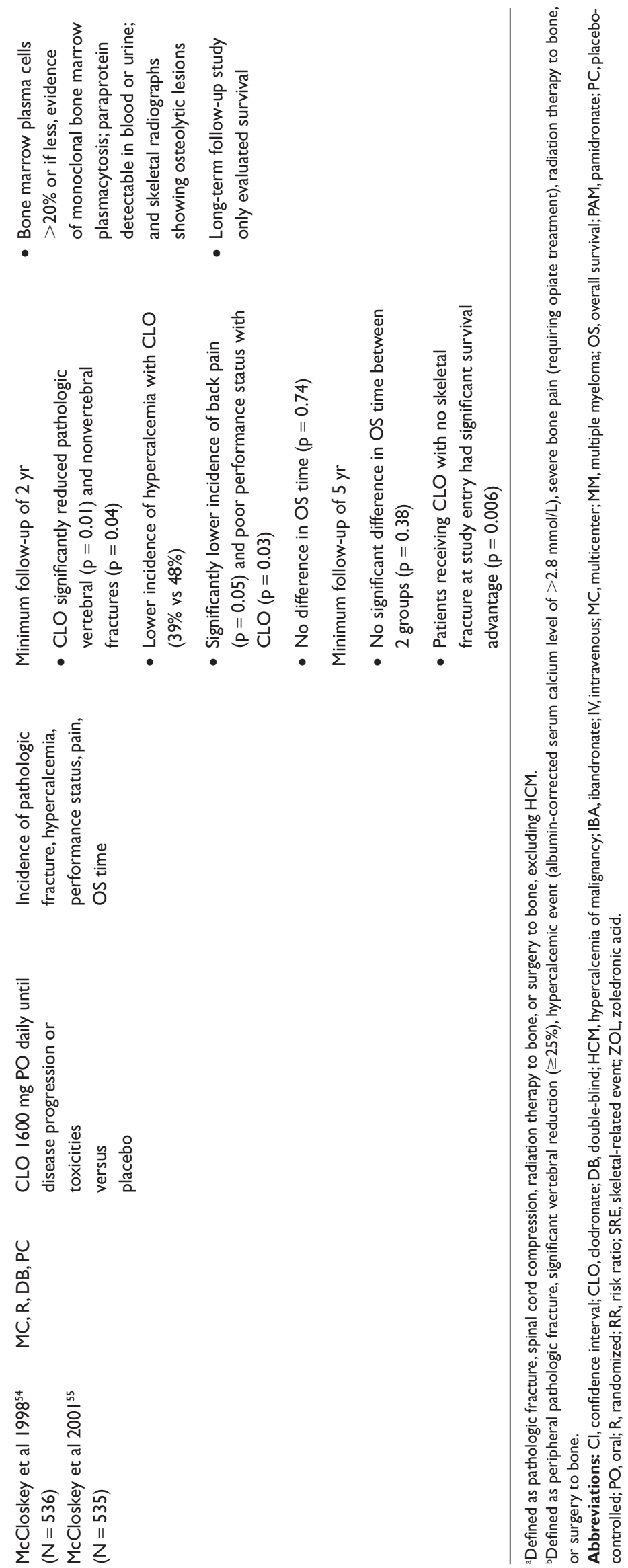

Drug Design, Development and Therapy 2009:3 
at least 2 hours or zoledronic acid $4 \mathrm{mg}$ IV delivered over 15 minutes every 3 to 4 weeks for a period of 2 years. IV or oral clodronate is an alternative in other countries, but it is not commercially available in the United States.

\section{Duration of therapy}

A consensus has not been reached regarding the appropriate duration of bisphosphonate therapy. Most studies of bisphosphonates in cancer patients with bone metastases did not treat patients beyond 2 years. However, ASCO has tried to place some clarity on the issue in both their clinical practice guidelines for patients with breast cancer and MM. ${ }^{57,66}$ In patients with breast cancer metastatic to bone, ASCO advises to continue bisphosphonates until evidence of a progressive decline in performance status develops, even in the presence of SREs. ${ }^{57}$ No evidence addressing the consequences of discontinuing bisphosphonate therapy after developing a SRE in breast cancer patients exists. Among patients with osteolytic metastases secondary to MM, 2 years of bisphosphonate therapy is recommended. ${ }^{66}$ After 2 years, treating physicians should consider treatment discontinuation if the MM is responding to therapy or is stable. Guidelines addressing duration of therapy in other solid tumors are not available.

\section{Bone turnover markers}

Investigators frequently assess markers of bone turnover as secondary end points in bisphosphonate studies. Biochemical markers of bone metabolism are indicative of either bone formation or bone resorption and may help identify patients likely to respond to and benefit from bisphosphonate therapy. ${ }^{67} \mathrm{~N}$-telopeptide of type I collagen (NTX) is of particular interest; patients with bone metastases and elevated NTX levels in urine have a significantly increased risk of SREs, disease progression, and death compared with patients with low NTX levels. ${ }^{68}$ In addition, urinary NTX normalization with pamidronate treatment has been linked with delays in bone lesion progression and a trend toward fewer fractures. ${ }^{69}$ Thus, NTX may be useful for monitoring therapeutic response to bisphosphonate therapy. However, because of the lack of sufficient, rigorous, prospective trials validating this approach, ASCO's clinical practice guidelines recommend that the use of these markers be confined to research protocols; currently, they should not be used in routine clinical practice..$^{57,66}$

\section{Conclusion}

Skeletal complications are a major source of cancer-related morbidity. In patients with bone metastases, bisphosphonates have become the standard of care for preventing or delaying SREs. In patients with breast cancer or MM involving bone, zoledronic acid and pamidronate were comparable in their ability to decrease the incidence of SREs and delay the onset of skeletal events. In patients with solid tumors (except breast cancer) that metastasize to the bone, only zoledronic acid has been proven effective; ibandronate is effective in colorectal cancer. Despite their impressive efficacy in the prevention of skeletal complications associated with malignancy, several questions related to bisphosphonate use remain. Studies are ongoing to evaluate the appropriate duration of therapy, validate the usefulness of bone markers in predicting response to therapy, understand management of toxicities such as osteonecrosis of the jaw, and determine the most appropriate and cost-effective time to initiate bisphosphonate therapy. ${ }^{70}$

\section{Acknowledgments}

The author thanks Kristin Hennenfent, PharmD, MBA, BCPS and Terri Davidson, PharmD, BCOP, who assisted with writing and editorial services, respectively, and Novartis Pharmaceuticals Corporation, who sponsored development of this article.

\section{Disclosures}

The author has no conflicts of interest to disclose.

\section{References}

1. Sambrook P, Cooper C. Osteoporosis. Lancet. 2006;367(9527): 2010-2018.

2. Hodgson SF, Watts NB; for AACE Osteoporosis Task Force. American Association of Clinical Endocrinologists medical guidelines for clinical practice for the prevention and treatment of postmenopausal osteoporosis: 2001 edition, with selected updates for 2003. Endocr Prac. 2003;9(6):544-564.

3. Coleman RE. Bisphosphonates: clinical experience. Oncologist. 2004;9(suppl 4):14-27.

4. Actonel [package insert]. Cincinnati, OH: Proctor and Gamble Pharmaceuticals, Incorporated; 2008.

5. Actonel plus calcium [package insert]. Cincinnati, $\mathrm{OH}$ : Proctor \& Gamble Pharmaceuticals, Incorporated; 2008.

6. Boniva Injection [package insert]. Nutley, NJ; Roche Pharmaceuticals; 2006.

7. Boniva Tablets [package insert]. Nutley, NJ; Roche Pharmaceuticals; 2006.

8. Fosamax [package insert]. Whitehouse Station, NJ; Merck \& Co; 2008.

9. Fosamax plus D [package insert]. Whitehouse Station, NJ; Merck \& Co; 2008.

10. Reclast [package insert]. East Hanover, NJ; Novartis Pharmaceuticals Corporation; 2008.

11. Black DM, Delmas PD, Eastell R, et al. Once-yearly zoledronic acid for treatment of postmenopausal osteoporosis. $N$ Engl J Med. 2007;356(18):1809-1822.

12. Lyles KW, Colón-Emeric CS, Magaziner JS, et al; HORIZON Recurrent Fracture Trial. Zoledronic acid and clinical fracture and mortality after hip fracture. N Engl J Med. 2007;357(18);1799-1809. 
13. Major P, Lortholary A, Hon J, et al. Zoledronic acid is superior to pamidronate in the treatment of hypercalcemia of malignancy: a pooled analysis of two randomized, controlled clinical trials. J Clin Oncol. 2001;19(2):558-567.

14. Lipton A. Treatment of bone metastases and bone pain with bisphosphonates. Support Cancer Ther. 2007;4(2):92-100.

15. Aapro M, Abrahamsson PA, Body JJ, et al. Guidance on the use of bisphosphonates in solid tumours: recommendations of an international expert panel. Ann Oncol. 2008;19(3):420-432.

16. Delea $\mathrm{T}$, Langer $\mathrm{C}, \mathrm{McKiernan} \mathrm{J}$, et al. The cost of treatment of skeletalrelated events in patients with bone metastases from lung cancer. Oncology. 2004;67(5-6):390-396.

17. Coleman RE. Clinical features of metastatic bone disease and risk of skeletal morbidity. Clin Cancer Res. 2006;12(20 Pt 2):6243s-6249s.

18. Mundy GR. Mechanisms of bone metastasis. Cancer. 1997;80(8): $1546-1556$.

19. Coleman R, Brown J, Terpos E, et al. Bone markers and their prognostic value in metastatic bone disease: clinical evidence and future directions. Cancer Treat Rev. 2008;34(7):629-639.

20. Guise TA, Mohammad KS, Clines G, et al. Basic mechanisms responsible for osteolytic and osteoblastic bone metastases. Clin Cancer Res. 2006;12(20 Pt 2):6213s-6216s.

21. Berenson JR, Rajdev L, Broder M. Treatment strategies for skeletal complications of cancer. Cancer Biol Ther. 2006;5(9):1074-1077.

22. Russell RG. Bisphosphonates: mode of action and pharmacology. Pediatrics. 2007;119:S150-S162.

23. Epstein S. Update of current therapeutic options for the treatment of postmenopausal osteoporosis. Clin Ther. 2006;28(2):151-173.

24. Roelofs AJ, Thompson K, Gordon S, Rogers MJ. Molecular mechanisms of action of bisphosphonates: current status. Clin Cancer Res. 2006;12(20, pt 2):6222s-6230s.

25. Zometa [package insert]. East Hanover, NJ; Novartis Pharmaceuticals Corporation; 2008

26. Aredia [package insert]. East Hanover, NJ; Novartis Pharmaceuticals Corporation; 2007.

27. European public assessment report: bondronat. European Medicines Agency Web site. Available at: http://www.emea.europa.eu/humandocs/ Humans/EPAR/bondronat/bondronat.htm. Accessed August 17, 2008.

28. European public assessment report: zometa. European Medicines Agency Web site. Available at: http://www.emea.europa.eu/humandocs/ Humans/EPAR/zometa/zometa.htm. Accessed August 16, 2008.

29. Johnson JR, Williams G, Pazdur R. End points and United States Food and Drug Administration approval of oncology drugs. J Clin Oncol. 2003;21(7):1404-1411.

30. Rosen LS, Gordon D, Kaminski M, et al. Zoledronic acid versus pamidronate in the treatment of skeletal metastases in patients with breast cancer or osteolytic lesions of multiple myeloma: a phase III, double-blind, comparative trial. Cancer J. 2001;7(5):377-387.

31. Rosen LS, Gordon D, Kaminski M, et al. Long-term efficacy and safety of zoledronic acid compared with pamidronate disodium in the treatment of skeletal complications in patients with advanced multiple myeloma or breast carcinoma: a randomized, double-blind, multicenter, comparative trial. Cancer. 2003;98(8):1735-1744.

32. Rosen LS, Gordon DH, Dugan W Jr, et al. Zoledronic acid is superior to pamidronate for the treatment of bone metastases in breast carcinoma patients with at least one osteolytic lesion. Cancer. 2004;100(1):36-43.

33. Kohno N, Aogi K, Minami H, et al. Zoledronic acid significantly reduces skeletal complications compared with placebo in Japanese women with bone metastases from breast cancer: a randomized, placebo-controlled trial. J Clin Oncol. 2005;23(15):3314-3321.

34. Carteni G, Bordonaro R, Giotta F, et al. Efficacy and safety of zoledronic acid in patients with breast cancer metastatic to bone: a multicenter clinical trial. Oncologist. 2006;11(7):841-848.

35. Hortobagyi GN, Theriault RL, Porter L, et al; for the Protocol 19 Aredia Breast Cancer Study Group. Efficacy of pamidronate in reducing skeletal complications in patients with breast cancer and lytic bone metastases. N Engl J Med. 1996;335(24):1785-1791.
36. Hortobagyi GN, Theriault RL, Porter L, et al; for the Protocol 19 Aredia Breast Cancer Study Group. Efficacy of pamidronate in reducing skeletal complications in patients with breast cancer and lytic bone metastases. J Clin Oncol. 1998;16(6):2038-2044.

37. Theriault RL, Lipton A, Hortobagyi GN, et al. Pamidronate reduces skeletal morbidity in women with advanced breast cancer and lytic bone lesions: a randomized, placebo-controlled trial. Protocol 18 Aredia Breast Cancer Study Group. J Clin Oncol. 1999;17(3):846-854.

38. Lipton A, Theriault RL, Hortobagyi GN, et al. Pamidronate prevents skeletal complications and is effective palliative treatment in women with breast carcinoma and osteolytic bone metastases: long term follow-up of two randomized, placebo-controlled trials. Cancer. 2000;88(5):1082-1090.

39. Body JJ, Diel IJ, Lichinitser MR, et al. Intravenous ibandronate reduces the incidence of skeletal complications in patients with breast cancer and bone metastases. Ann Oncol. 2003;14(9):1399-1405.

40. Body JJ, Diel IJ, Lichinitzer M, et al. Oral ibandronate reduces the risk of skeletal complications in breast cancer patients with metastatic bone disease: results from two randomised, placebo-controlled phase III studies. Br J Cancer. 2004;90(6):1133-1137.

41. Paterson AH, Powles TJ, Kanis JA, McCloskey E, Hanson J, Ashley S. Double-blind controlled trial of oral clodronate in patients with bone metastases from breast cancer. J Clin Oncol. 1993;11(1):59-65.

42. Kristensen B, Ejlertsen B, Groenvold M, Hein S, Loft H, Mouridsen HT. Oral clodronate in breast cancer patients with bone metastases: a randomized study. J Intern Med. 1999;246(1):67-74.

43. Saad F, Gleason DM, Murray R, et al; for Zoledronic Acid Prostate Cancer Study Group. A randomized, placebo-controlled trial of zoledronic acid in patients with hormone-refractory metastatic prostate carcinoma. J Natl Cancer Inst. 2002;94(19):1458-1468.

44. Saad F, Gleason DM, Murray R, et al; for Zoledronic Acid Prostate Cancer Study Group. Long-term efficacy of zoledronic acid for the prevention of skeletal complications in patients with metastatic hormonerefractory prostate cancer. J Natl Cancer Inst. 2004;96(11):879-882.

45. Small EJ, Smith MR, Seaman JJ, Petrone S, Kowalski MO. Combined analysis of two multicenter, randomized, placebo-controlled studies of pamidronate disodium for the palliation of bone pain in men with metastatic prostate cancer. J Clin Oncol. 2003;21(23):4277-4284.

46. Dearnaley DP, Sydes MR, Mason MD, et al; for the MRC PR05 Collaborators. A double-blind, placebo-controlled, randomized trial of oral sodium clodronate for metastatic prostate cancer (MRC PR05 Trial). J Natl Cancer Inst. 2003;95(17):1300-1311.

47. Rosen LS, Gordon D, Tchekmedyian S, et al. Zoledronic acid versus placebo in the treatment of skeletal metastases in patients with lung cancer and other solid tumors: a phase III, double-blind, randomized trial - the Zoledronic Acid Lung Cancer and Other Solid Tumors Study Group. J Clin Oncol. 2003;21(16):3150-3157.

48. Rosen LS, Gordon D, Tchekmedyian NS, et al. Long-term efficacy and safety of zoledronic acid in the treatment of skeletal metastases in patients with nonsmall cell lung carcinoma and other solid tumors: a randomized, phase III, double-blind, placebo-controlled trial. Cancer. 2004;100(12):2613-2621.

49. Heras P, Karagiannis S, Kritikos K, Hatzopoulos A, Mitsibounas D. Ibandronate is effective in preventing skeletal events in patients with bone metastases from colorectal cancer. Eur J Cancer Care. 2007;16(6):539-542.

50. Piga A, Bracci R, Ferretti B, et al. A double blind randomized study of oral clodronate in the treatment of bone metastases from tumors poorly responsive to chemotherapy. J Exp Clin Cancer Res. 1998;17(2):213-217.

51. Berenson JR, Lichtenstein A, Porter L, et al; for Myeloma Aredia Study Group. Efficacy of pamidronate in reducing skeletal events in patients with advanced multiple myeloma. $N$ Engl J Med. 1996;334(8):488-493.

52. Berenson JR, Lichtenstein A, Porter L, et al; for the Myeloma Aredia Study Group. Long-term pamidronate treatment of advanced multiple myeloma patients reduces skeletal events. J Clin Oncol. 1998;16(2):593-602. 
53. Menssen HD, Sakalová A, Fontana A, et al. Effects of long-term intravenous ibandronate therapy on skeletal-related events, survival, and bone resorption markers in patients with advanced multiple myeloma. J Clin Oncol. 2002;20(9):2353-2359.

54. McCloskey EV, MacLennan ICM, Drayson MT, Chapman C, Dunn J, Kanis JA. A randomized trial of the effect of clodronate on skeletal morbidity in multiple myeloma. MRC Working Party on Leukaemia in Adults. Br J Haematol. 1996;100(2):317-325.

55. McCloskey EV, Dunn JA, Kanis JA, MacLennan IC, Drayson MT. Long-term follow-up of a prospective, double-blind, placebo-controlled randomized trial of clodronate in multiple myeloma. MRC Working Party on Leukaemia in Adults. Br J Haematol. 2001;113(4):1035-1043.

56. Major P, Lortholary A, Hon J, et al. Zoledronic acid is superior to pamidronate in the treatment of hypercalcemia of malignancy: a pooled analysis of two randomized, controlled clinical trials. J Clin Oncol. 2001;19(2):558-567.

57. Hilner BE, Ingle JN, Chlebowski RT, et al. American Society of Clinical Oncology 2003 update on the role of bisphosphonates and bone health issues in women with breast cancer. J Clin Oncol. 2003;21(21):4042-4057.

58. The NCCN Breast Cancer Clinical Practice Guidelines in Oncology (version 2.2008). (C) 2008 National Comprehensive Cancer Network, Inc. Available at: http://www.nccn.org. Accessed July 14, 2008.

59. Pavlakis N, Schmidt R, Stockler M. Bisphosphonates for breast cancer. Cochrane Database Syst Rev. 2005;20(3):CD003474.

60. The NCCN Prostate Cancer Clinical Practice Guidelines in Oncology (version 1.2008). (C) 2008 National Comprehensive Cancer Network, Inc. Available at: http://www.nccn.org. Accessed July 14, 2008.

61. Yuen KK, Shelley M, Sze WM, Wilt T, Mason MD. Bisphosphonates for advanced prostate cancer. Cochrane Database Syst Rev. 2006;4: CD006250.

62. Hoosier Oncology Group. Phase III, randomized, double-blind, placebocontrolled trial evaluating the ability of risedronate to prevent skeletal related events in patients with metastatic prostate cancer commencing hormonal therapy: Hoosier Oncology Group GU02-41. ClinicalTrials. gov. http://www.clinicaltrials.gov/ct2/show/NCT00216060?term= risedronate+prostate\&rank=2. Accessed July 15, 2008.
63. Zoledronate in preventing skeletal (bone)-related events in patients who are receiving androgen deprivation therapy for prostate cancer and bone metastases. ClinicalTrials.gov Web site. http://www.clinicaltrials. gov/ct2/show/NCT00698308?term=NCT00698308\&rank=1. Accessed July 15,2008

64. The NCCN Non-Small Cell Lung Cancer Clinical Practice Guidelines in Oncology (version 1.2009). (C) 2009 National Comprehensive Cancer Network, Inc. Available at: http://www.nccn.org. Accessed October 1, 2008.

65. Zoledronic acid treatment (every 4 or 12 weeks) to prevent skeletal complications in advanced multiple myeloma patients (Z-MARK). ClinicalTrials.gov Web site. http://www.clinicaltrials.gov/ct2/show/ NCT00622505?term=zoledronic+acid+multiple+myeloma\&rank=4. Accessed August 16, 2008.

66. Kyle RA, Yee GC, Somerfield MR, et al. American Society of Clinical Oncology 2007 clinical practice guideline update on the role of bisphosphonates in multiple myeloma. J Clin Oncol. 2007;25(17):2464-2472.

67. Clemons M, Dranitsaris G, Cole D, Gainford MC. Too much, too little, too late to start again? Assessing the efficacy of bisphosphonates in patients with bone metastases from breast cancer. Oncologist. 2006;11(3):227-233.

68. Brown JE, Cook RJ, Major P, et al. Bone turnover markers as predictors of skeletal complications in prostate cancer, lung cancer, and other solid tumors. J Natl Cancer Inst. 2005;97(1):59-69.

69. Lipton A, Demers L, Curley E, et al. Markers of bone resorption in patients treated with pamidronate. Eur J Cancer. 1998;34(13):2021-2026.

70. ClinicalTrials.gov Web site. http://www.clinicaltrials.gov. Accessed October 14, 2008. 